\title{
Power Allocation for Energy-Harvesting-based Fading Cognitive Multiple Access Channels: with or without Successive Interference Cancellation
}

\author{
Qun Li and Ding Xu
}

\begin{abstract}
This paper considers a fading cognitive multiple access channel (CMAC), where multiple secondary users (SUs), who share the spectrum with a primary user (PU), transmit to a cognitive base station (CBS). A power station is assumed to harvest energy from the nature and then provide power to the SUs. We investigate the power allocation problems for such a CMAC to maximize the SU sum rate under the interference power constraint, the sum transmit power constraint and the peak transmit power constraint of each individual SU. In particular, two scenarios are considered: with successive interference cancellation (SIC) and without SIC. For the first scenario, the optimal power allocation algorithm is derived. For the second scenario, a heuristic algorithm is proposed. We show that the proposed algorithm with SIC outperforms the algorithm without SIC in terms of the SU sum rate, while the algorithm without SIC outperforms the algorithm with SIC in terms of the number of admitted SUs for a high sum transmit power limit and a low peak transmit power limit of each individual SU.
\end{abstract}

Keywords-Cognitive radio, power allocation, multiple access channels, energy harvesting

\section{INTRODUCTION}

In recent years, various wireless multimedia services have been increasingly popular, which causes huge demand for radio spectrum resources. However, most radio spectrum resources have been allocated to various wireless services. This poses a big challenge to the spectrum allocation regulation. In this respect, many field spectrum usage measurements show that most of the allocated spectrum is under-utilized. For example, in [1], it is shown that the average spectrum usage is approximately $15.2 \%$ in Beijing, China, for spectrum bands from $440 \mathrm{MHz}$ to $2700 \mathrm{MHz}$. Thus, it is urgent to improve the spectrum utilization rather than finding more spectrum resources. In this regard, cognitive radio (CR) [2], is a promising solution to solve the spectrum scarcity problem by allowing secondary users (SUs) to utilize the spectrum allocated to primary users (PUs). A very popular model in $\mathrm{CR}$ is spectrum sharing which limits the interference power from the SUs to the PUs.

Power allocation is very important for controlling the interference to the PUs. Subject to the interference power constraint and the transmit power constraint, the power allocation problems for a fading $\mathrm{CR}$ point to point channel to maximize the

This work was supported by the National Natural Science Foundation of China (Grant No. 61401218) and NUPTSF (Grant No. NY214062 and NY213086).

Authors are with Wireless Communication Key Lab of Jiangsu Province, Nanjing University of Posts and Telecommunications, Nanjing 210003, China, e-mail: liqun@njupt.edu.cn, xuding.bupt@gmail.com. Ding Xu is the corresponding author ergodic capacity and the outage capacity were investigated in [3]. In [4], under the same system model as in [3], we proposed power allocation algorithms that can balance between the goal of maximizing the ergodic capacity and the goal of minimizing the outage probability. Besides the $\mathrm{CR}$ point to point channel model investigated in current studies such as [3], [4], a more complicated cognitive multiple access channel (CMAC) model has also attracted a lot of attention. In [5], the power allocation problems for maximizing the ergodic capacity of the fading CMAC under various power constraints were investigated. In [6], the optimal power allocation to achieve the outage capacity region boundary for the fading CMAC was derived. In [7], the power allocation problem for the fading CMAC to maximize the ergodic capacity was reformulated as a geometrical problem and the optimal power allocation was then derived. In [8], the optimal power allocation for an interference-limited CMAC to minimize the outage probability of the SUs was proposed. In [9], the weighted sum rate maximization power allocation problem for a CMAC was studied. All these works in [5]-[9] assumed that successive interference cancellation (SIC) is available and there is no mutual interference between the SUs. For the case that SIC is unavailable, [10] derived the optimal power allocation strategies to maximize the ergodic capacity and the outage capacity for a two-user CMAC under the interference power constraint, [11] proposed a greedy power allocation policy to achieve the effective capacity region boundary for a fading CMAC under the interference power constraint, [12] investigated the power allocation problems to maximize the ergodic sum rate for a fading CMAC under various power constraints, and [13] proposed two heuristic power allocation algorithms to maximize the ergodic sum rate of a fading CMAC under the PU outage constraint.

Different from the previous works in investigating power allocation problem in CMAC, this paper studies the power allocation problem for a fading CMAC based on energy harvesting to maximize the sum rate of the SUs with or without SIC. Energy harvesting is a promising approach to decrease the energy cost in wireless networks. We consider a scenario where a power station harvests energy from the environments and then provides power to the SUs. In this scenario, since the harvested energy is shared among the SUs, a sum transmit power constraint for the SUs is introduced. It is noted that such constraint was not considered in the previous works such as [5]-[13]. The related works in energy-harvestingbased CR networks can be found in [14]-[16]. However, to our best knowledge, the scenario of energy-harvesting-based 
CMAC considered in this paper has not been considered yet in literature.

The main contributions and the key findings of this paper are listed below:

- The power allocation problems for the SUs to maximize the SU sum rate for the fading energy-harvesting-based CMAC under the interference power constraint, the sum transmit power constraint and the peak transmit power constraint of each individual SU are investigated considering two scenarios: (1) with SIC ; (2) without SIC.

- We derive the optimal power allocation algorithm for the first scenario. For the second scenario, a heuristic power allocation algorithm is proposed.

- It is shown that, although the proposed power allocation algorithm with SIC always achieves higher SU sum rate than that of the algorithm without SIC, the number of admitted SUs achieved by the algorithm without SIC is higher than that achieved by the algorithm with SIC for a high sum transmit power limit and a low peak transmit power limit of each individual SU. It is also shown that the number of admitted SUs increases as the peak transmit power limit of each individual SU decreases.

The rest of the paper is organized as follows. The system model is described in Section II. The power allocation problems to maximize the SU sum rate for the fading energyharvesting-based CMAC with SIC and without SIC are investigated in Section III and Section IV, respectively. Section $\mathrm{V}$ gives simulation results to verify the proposed algorithms. Section VI concludes the paper.

\section{SYSTEM MODEL}

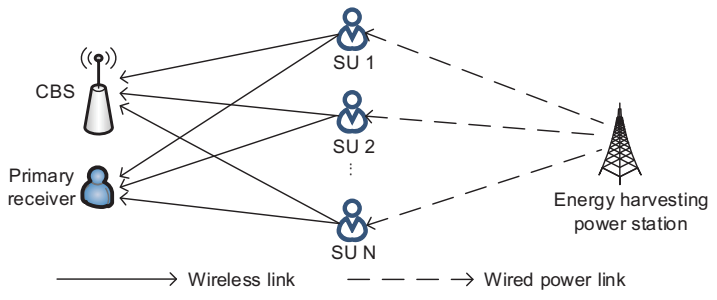

Fig. 1. System model.

We consider a CMAC as shown in Fig. 1, which consists of one receiving cognitive base station (CBS), $N$ SUs, and one power station. The power station continuously harvest energy from nature, and then transfer the energy to the SUs through wired power lines ${ }^{1}$. It is assumed that the CMAC shares the same narrow band with the PU. Block-fading channel model is assumed for all the channels involved, i.e., the channels keep constant in one transmission block, but may change from

${ }^{1}$ It is noted that using an energy harvesting power station to provide energy to the SUs is suitable for the scenarios when the SUs are not mobile and fixed at some locations. If energy harvesting is performed at the SUs, the amount of harvested energy is limited due to limited capability of the SUs. Thus, using a more powerful energy harvesting power station can provide more harvested energy to the SUs. Besides, a central power station provides more flexible and efficient power allocation compared to distributed energy supply. one transmission block to another. The instantaneous channel power gains from $\mathrm{SU} i$ to $\mathrm{CBS}$ and $\mathrm{SU} i$ to the $\mathrm{PU}$ receiver are denoted by $h_{i}$ and $g_{i}$, respectively. We assume that the SUs and the power station are not equipped with rechargeable batteries, and the total power harvested and available for the SUs for one transmission block is $\bar{P}$. Note that the value of $\bar{P}$ changes from one block to another. The noise power is denoted by $\sigma^{2}$.

Let $\mathbf{p}=\left[p_{1}, \ldots, p_{N}\right]$ denote the power allocation vector of the SUs, where $p_{i}$ denote the transmit power of SU $i$. The interference power constraint is adopted to protect the $\mathrm{PU}$ transmission as given by

$$
\sum_{i=1}^{N} p_{i} g_{i} \leq \bar{Q}
$$

where $\bar{Q}$ is the predefined interference power limit. The sum transmit power of the SUs can not be higher than the power available at the power station, as given $b y^{2}$

$$
\sum_{i=1}^{N} p_{i} \leq \bar{P}
$$

Besides, the transmit power of each individual SU is restricted due to hardware limit as

$$
p_{i} \leq P_{i}^{\max }, i=1, \ldots, N,
$$

where $P_{i}^{\max }$ denotes the peak transmit power limit of SU $i$.

With SIC capability at the CBS, the sum rate of the SUs is given by

$$
R(\mathbf{p})=\ln \left(1+\frac{\sum_{i=1}^{N} p_{i} h_{i}}{\sigma^{2}}\right) .
$$

On the other hand, the sum rate of the SUs without SIC capability at the CBS is given by

$$
R(\mathbf{p})=\sum_{i=1}^{N} \ln \left(1+\frac{p_{i} h_{i}}{\sigma^{2}+\sum_{j=1, j \neq i}^{N} p_{j} h_{j}}\right) .
$$

\section{POWER ALLOCATION WITH SIC}

This section investigates the problem of power allocation for the SUs to maximize their sum rate with SIC capability at the CBS. The problem is formulated as follows

$$
\begin{aligned}
\mathrm{P} 1: \max _{\boldsymbol{p}} & \ln \left(1+\frac{\sum_{i=1}^{N} p_{i} h_{i}}{\sigma^{2}}\right) \\
\text { s.t. } & \sum_{i=1}^{N} p_{i} g_{i} \leq \bar{Q}, \\
& \sum_{i=1}^{N} p_{i} \leq \bar{P}, \\
& p_{i} \leq P_{i}^{\max }, i=1, \ldots, N \\
& p_{i} \geq 0, i=1, \ldots, N
\end{aligned}
$$

${ }^{2}$ It is noted that the sum transmit power constraint in (2) is applied due to introducing the central power station. Compared to distributed energy supply which does not require the constraint in (2), the sum transmit power constraint leads to more complicated power allocation as the transmit powers of the SUs are coupled in (2). 
It is easy to verify that the objective function in (6) is concave with respect to $\boldsymbol{p}$ and thus $\mathrm{P} 1$ can be solved by the convex optimization [17]. The Lagrangian of P1 can be written as

$$
\begin{gathered}
L(\lambda, \mu, \boldsymbol{\nu}, \boldsymbol{\varepsilon}, \boldsymbol{p})=\ln \left(1+\frac{\sum_{i=1}^{N} p_{i} h_{i}}{\sigma^{2}}\right)-\lambda\left(\sum_{i=1}^{N} p_{i} g_{i} \leq \bar{Q}\right) \\
-\mu\left(\sum_{i=1}^{N} p_{i}-\bar{P}\right)-\sum_{i=1}^{N} \nu_{i}\left(p_{i}-P_{i}^{\max }\right)+\sum_{i=1}^{N} \varepsilon_{i} p_{i}
\end{gathered}
$$

where $\lambda, \mu, \nu_{i}$ and $\varepsilon_{i}$ are the non-negative dual variables associated with the constraints in (7), (8), (9) and (10), respectively, $i=1, \ldots, N$, with $\boldsymbol{\nu}=\left[\nu_{1}, \ldots, \nu_{N}\right]$ and $\varepsilon=\left[\varepsilon_{1}, \ldots, \varepsilon_{N}\right]$. According to the KKT conditions [17], the optimal solutions of P1 shall satisfy the following equations

$$
\begin{aligned}
\frac{h_{i}}{\sigma^{2}+\sum_{n=1}^{N} p_{n} h_{n}}-\lambda g_{i}-\mu-\nu_{i}+\varepsilon_{i} & =0, \forall i \\
\nu_{i}\left(p_{i}-P_{i}^{\max }\right) & =0, \forall i \\
\nu_{i} & \geq 0, \forall i \\
p_{i} & \leq P_{i}^{\max }, \forall i \\
\varepsilon_{i} p_{i} & =0, \forall i \\
\varepsilon_{i} & \geq 0, \forall i \\
p_{i} & \geq 0 . \forall i
\end{aligned}
$$

From the above KKT optimality conditions, we can obtain the following lemma:

Lemma 1: The solution of $\mathrm{P} 1$ has at most one SU's transmit power, denoted by $p_{i}$ that satisfies $0<p_{i}<P_{i}^{\max }$.

Proof: Supposing that there are two SUs $i$ and $j$ with $0<$ $p_{i}<P_{i}^{\max }$ and $0<p_{j}<P_{j}^{\max }$, then, from (13), (14), (16), (17), we have $\nu_{i}=\nu_{j}=0$ and $\varepsilon_{i}=\varepsilon_{j}=0$. Using these results, from (12), we have

$$
\begin{gathered}
\frac{h_{i}}{\lambda g_{i}+\mu}=\sigma^{2}+\sum_{n=1}^{N} p_{n} h_{n}, \\
\frac{h_{j}}{\lambda g_{j}+\mu}=\sigma^{2}+\sum_{n=1}^{N} p_{n} h_{n} .
\end{gathered}
$$

Then, from the above two equations, we have

$$
\frac{h_{i}}{\lambda g_{i}+\mu}=\frac{h_{j}}{\lambda g_{j}+\mu} \text {. }
$$

Since $\left\{h_{i}, i=1, \ldots, N\right\}$ and $\left\{g_{i}, i=1, \ldots, N\right\}$ are sets of independent random variables and $\mu$ is constant, the probability of satisfying the above equality is zero. Thus, it can be concluded that there is at most one $\mathrm{SU} i$ with $0<p_{i}<P_{i}^{\max }$.

Lemma 1 is thus proved.

Lemma 1 indicates that most of $p_{i}, i=1, \ldots, N$ is either 0 or $P_{i}^{\max }$. The following lemma shows the relationship between the SU whose transmit power is zero and the SU whose transmit power is nonzero.

Lemma 2: Supposing that any two SUs, $i$ and $j$ satisfy $p_{i}=$ 0 and $p_{j}>0$, then the following inequality must be satisfied:

$$
\frac{h_{i}}{\lambda g_{i}+\mu} \leq \frac{h_{j}}{\lambda g_{j}+\mu} \text {. }
$$

Proof: Supposing that any two arbitrary SUs $i$ and $j$ with $p_{i}=0$ and $p_{j}>0$, from (13), (14), (16), (17) we have $\nu_{i}=0$ and $\varepsilon_{j}=0$. Then, from (12), we have

$$
\begin{aligned}
\frac{h_{i}}{\lambda g_{i}+\mu-\varepsilon_{i}} & =\sigma^{2}+\sum_{n=1}^{N} p_{n} h_{n}, \\
\frac{h_{j}}{\lambda g_{j}+\mu+\nu_{j}} & =\sigma^{2}+\sum_{n=1}^{N} p_{n} h_{n} .
\end{aligned}
$$

From the above two equations and using the fact that $\varepsilon_{i} \geq 0$ and $\nu_{j} \geq 0$, we get

$$
\frac{h_{i}}{\lambda g_{i}+\mu} \leq \frac{h_{j}}{\lambda g_{j}+\mu} .
$$

Lemma 2 is thus proved.

Lemma 3: Supposing that any two SUs, $i$ and $j$ satisfy $0<$ $p_{i}<P_{i}^{\max }$ and $p_{j}=P_{j}^{\max }$, then the following inequality must be satisfied:

$$
\frac{h_{i}}{\lambda g_{i}+\mu} \leq \frac{h_{j}}{\lambda g_{j}+\mu}
$$

Proof: Supposing that any two arbitrary SUs $i$ and $j$ with $0<p_{i}<P_{i}^{\max }$ and $p_{j}=P_{j}^{\max }$, from (13), (14), (16), (17) we have $\nu_{i}=0, \varepsilon_{i}=0$ and $\varepsilon_{j}=0$. Then, from (12), we have

$$
\begin{aligned}
\frac{h_{i}}{\lambda g_{i}+\mu} & =\sigma^{2}+\sum_{n=1}^{N} p_{n} h_{n}, \\
\frac{h_{j}}{\lambda g_{j}+\mu+\nu_{j}} & =\sigma^{2}+\sum_{n=1}^{N} p_{n} h_{n} .
\end{aligned}
$$

Using the fact that $\nu_{j} \geq 0$, we get

$$
\frac{h_{i}}{\lambda g_{i}+\mu} \leq \frac{h_{j}}{\lambda g_{j}+\mu} \text {. }
$$

Lemma 3 is thus proved.

Without loss of generality, we assume that the SUs are sorted such that $\frac{h_{1}}{\lambda g_{1}+\mu} \geq \frac{h_{2}}{\lambda g_{2}+\mu} \geq \ldots \geq \frac{h_{N}}{\lambda g_{N}+\mu}$. Assuming that $K$ SUs can transmit with nonzero power, from Lemma 1 , Lemma 2 and Lemma 3 we have $p_{i}=p_{i}^{\max }, i=1, \ldots, K-1$ and $p_{j}=0, j=K+1, \ldots, N$. The transmit power of SU $K$ satisfies $0<p_{K} \leq p_{K}^{\max }$. The following lemma gives the expression of $p_{K}$ and the way to obtain the value of $K$.

Lemma 4: The transmit power of SU $K$ is

$p_{K}=\min \left(p_{K}^{\max }, \frac{1}{h_{K}}\left(\frac{h_{K}}{\lambda g_{K}+\mu}-\sigma^{2}-\sum_{n=1}^{K-1} p_{n}^{\max } h_{n}\right)\right)$.

The value of $K$ is the largest value of $k$ such that

$$
\sum_{n=1}^{k-1} p_{n}^{\max } h_{n}<\frac{h_{k}}{\lambda g_{k}+\mu}-\sigma^{2}
$$

Proof: Supposing that $0<p_{K}<p_{K}^{\max }$, then, from (13), (14), (16), (17), we have $\nu_{K}=0$ and $\varepsilon_{K}=0$. Inserting these results into (12), we have

$$
\frac{h_{K}}{\lambda g_{K}+\mu}=\sigma^{2}+\sum_{n=1}^{N} p_{n} h_{n}
$$


Using $p_{i}=p_{i}^{\max }, i=1, \ldots, K-1$ and $p_{j}=0, j=K+$ $1, \ldots, N$, we can rewrite the above expression as

$$
\frac{h_{K}}{\lambda g_{K}+\mu}=\sigma^{2}+\sum_{n=1}^{K-1} p_{n}^{\max } h_{n}+p_{K} h_{K},
$$

from which we have

$$
p_{K}=\frac{1}{h_{K}}\left(\frac{h_{K}}{\lambda g_{K}+\mu}-\sigma^{2}-\sum_{n=1}^{K-1} p_{n}^{\max } h_{n}\right) .
$$

Since $p_{K} \leq p_{K}^{\max }$, it then follows that

$p_{K}=\min \left(p_{K}^{\max }, \frac{1}{h_{K}}\left(\frac{h_{K}}{\lambda g_{K}+\mu}-\sigma^{2}-\sum_{n=1}^{K-1} p_{n}^{\max } h_{n}\right)\right)$.

Since $p_{K}>0$, from (34), we have

$$
\sum_{n=1}^{K-1} p_{n}^{\max } h_{n}<\frac{h_{K}}{\lambda g_{K}+\mu}-\sigma^{2} .
$$

For SUs $j=K+1, \ldots, N$, since $p_{j}=0$ and $\nu_{j}=0$, from (12), we have

$$
\frac{h_{j}}{\lambda g_{j}+\mu-\varepsilon_{j}}=\sigma^{2}+\sum_{n=1}^{K-1} p_{n}^{\max } h_{n}+p_{K} h_{k} .
$$

Since $p_{K} \leq P_{K}^{\max }$ and $p_{j}=0, j=K+1, \ldots, N$, we have

$$
\frac{h_{j}}{\lambda g_{j}+\mu-\varepsilon_{j}}-\sigma^{2} \leq \sum_{n=1}^{j-1} p_{n}^{\max } h_{n} .
$$

Consider the fact that $\varepsilon_{j} \geq 0$, we have

$$
\frac{h_{j}}{\lambda g_{j}+\mu}-\sigma^{2} \leq \sum_{n=1}^{j-1} p_{n}^{\max } h_{n}
$$

The above inequality indicates that any $\mathrm{SU} j \in\{K+$ $1, \ldots, N\}$ does not satisfies the inequality $\sum_{n=1}^{j-1} p_{n}^{\max } h_{n}<$ $\frac{h_{j}}{\lambda g_{j}+\mu}-\sigma^{2}$. Thus, From (36), we conclude that the value of $K$ is the largest value of $k$ that satisfies the inequality in (31).

Lemma 4 is thus proved.

Finally, the values of $\lambda$ and $\mu$ can be obtained by the subgradient-based method. Our proposed optimal solution to P1 is summarized in Algorithm 1.

Remark 1: In the proof of Lemma 1, we assume that $\frac{h_{i}}{\lambda g_{i}+\mu}=\frac{h_{j}}{\lambda g_{j}+\mu}$ is impossible for arbitrary $i \in\{1, \ldots, N\}$ and $j \in\{1, \ldots, N\}, j \neq i$ due to the fact that the probability of satisfying the equality is zero. However, for extreme case when the equality holds, Lemma 1 will not be tenable. In such extreme case, Lemma 2 and Lemma 3 still hold, and in Lemma 4 , it is easy to verify that the value of $K$ is still the largest value of $k$ such that the inequality in (31) is satisfied. Thus, based on above discussions, if $\frac{h_{K-1}}{\lambda g_{K-1}+\mu}=\frac{h_{K}}{\lambda g_{K}+\mu}$ does not hold, then Lemma 4 still establishes and Algorithm 1 does not need to be modified. Otherwise, if there exists a $M(1 \leq M<K)$ that satisfies the equality $\frac{h_{M}}{\lambda g_{M}+\mu}=\frac{h_{M+1}}{\lambda g_{M+1}+\mu} \ldots=\frac{h_{K}}{\lambda g_{K}+\mu}$,

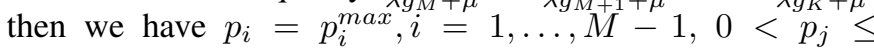
$p_{j}^{\max }, j=M, \ldots, K$ and $p_{k}=0, k=K+1, \ldots, N$. Thus, in this case, we need to determine $p_{j}, j=M, \ldots, K$, and we propose a heuristic scheme as follows. First, we let

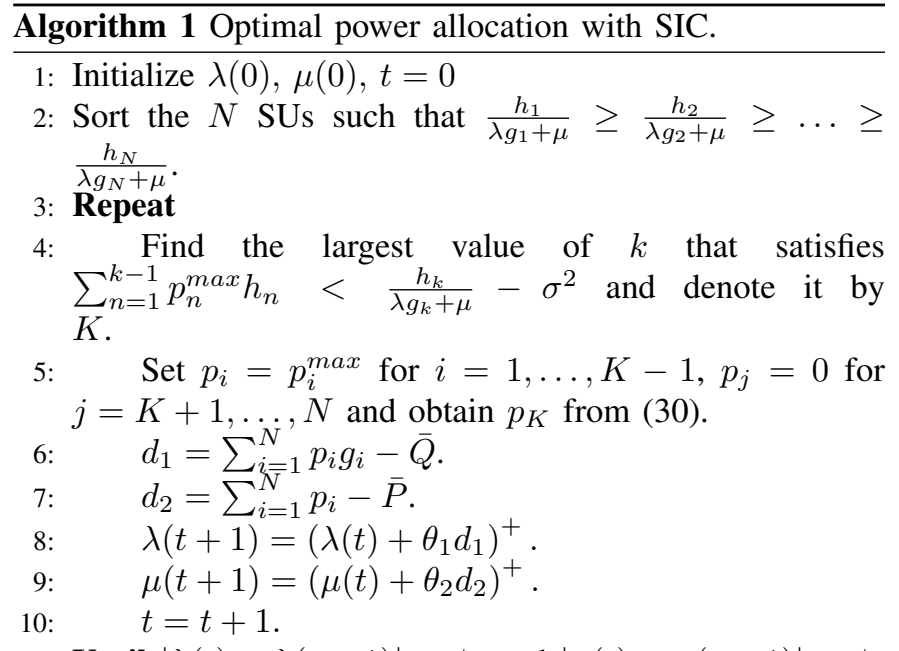

11: Until $|\lambda(t)-\lambda(t-1)| \leq \zeta_{1}$ and $|\mu(t)-\mu(t-1)| \leq \zeta_{2}$ are satisfied simultaneously.

where $t$ is the iteration number, $\theta_{1}$ and $\theta_{2}$ are scalar step sizes, $\zeta_{1}$ and $\zeta_{2}$ denote the error tolerances.

$p_{j}=p_{j}^{\max }, j=M, \ldots, K-1$ and calculate $p_{K}$ from (30). If $p_{K}$ satisfies $0<p_{k} \leq p_{K}^{\max }$, then we have obtained $p_{j}, j=M, \ldots, K$. Otherwise, we iteratively decrease $p_{j}, j=$ $M, \ldots, K-1$ as $p_{j}=\pi p_{j}, j=M, \ldots, K-1$ until $p_{k}=$ $\frac{1}{h_{K}}\left(\frac{h_{K}}{\lambda g_{K}+\mu}-\sigma^{2}-\sum_{n=1}^{M-1} p_{n}^{\max } h_{n}-\sum_{n=M}^{K-1} p_{n} h_{n}\right)>0$, where $\pi$ is a bit less than 1 .

\section{Power Allocation Without SIC}

This section investigates the problem of power allocation for the SUs to maximize their sum rate without SIC capability at the CBS. The problem is formulated as

$$
\begin{aligned}
\mathrm{P} 2: \max _{\boldsymbol{p}} & \sum_{i=1}^{N} \ln \left(1+\frac{p_{i} h_{i}}{\sigma^{2}+\sum_{j=1, j \neq i}^{N} p_{j} h_{j}}\right) \\
\text { s.t. } & \sum_{i=1}^{N} p_{i} g_{i} \leq \bar{Q}, \\
& \sum_{i=1}^{N} p_{i} \leq \bar{P} \\
& p_{i} \leq P_{i}^{\max }, i=1, \ldots, N \\
& p_{i} \geq 0, i=1, \ldots, N
\end{aligned}
$$

It is easy to observe that the objective function in (40) is nonconcave and thus P2 can not be solved by convex optimization. To solve the above problem, we introduce the following lemmas:

Lemma 5: At least one of the constraints in (41)-(44) is satisfied with equality for the optimal solution of $\mathrm{P} 2$.

Proof: Denote $f(\boldsymbol{p})=\sum_{i=1}^{N} \ln \left(1+\frac{p_{i} h_{i}}{\sigma^{2}+\sum_{j=1, j \neq i}^{N} p_{j} h_{j}}\right)$ as the objective function in (40). We prove the lemma by contradiction. We assume that all of the constraints in (41)(44) are satisfied with strict inequality for the optimal solution of P2. For an arbitrary $i \in\{1, \ldots, N\}$, the first derivative of 
$f(\boldsymbol{p})$ with respect to $p_{i}$ can be obtained as

$$
\begin{aligned}
& \frac{\partial f(\boldsymbol{p})}{\partial p_{i}}=\frac{1}{\left(1+\frac{p_{i} h_{i}}{\sigma^{2}+\sum_{j=1, j \neq i}^{N} p_{j} h_{j}}\right)} \times \frac{h_{i}}{\sigma^{2}+\sum_{j=1, j \neq i}^{N} p_{j} h_{j}} \\
& -\sum_{n=1, n \neq i}^{N} \frac{1}{1+\frac{p_{i} h_{i}}{\sigma^{2}+\sum_{j=1, j \neq n}^{N} p_{j} h_{j}}} \times \frac{p_{n} h_{n} h_{i}}{\left(\sigma^{2}+\sum_{j=1, j \neq n}^{N} p_{j} h_{j}\right)^{2}} \\
& =\frac{h_{i}}{\sigma^{2}+\sum_{j=1}^{N} p_{j} h_{j}}\left(1-\sum_{n=1, n \neq i}^{N} \frac{p_{n} h_{n} h_{i}}{\sigma^{2}+\sum_{j=1, j \neq n}^{N} p_{j} h_{j}}\right) .
\end{aligned}
$$

It is observed that $\frac{h_{i}}{\sigma^{2}+\sum_{j=1}^{N} p_{j} h_{j}}$ is strictly positive and $1-$ $\sum_{n=1, n \neq i}^{N} \frac{p_{n} h_{n} h_{i}}{\sigma^{2}+\sum_{j=1, j \neq n}^{N} p_{j} h_{j}}$ is a strictly increasing function of $p_{i}$. Therefore, $\frac{\partial f(\boldsymbol{p})}{\partial p_{i}}=0$ has one unique solution and is denoted as $p_{i}=x_{i}$. We discuss the optimal $p_{i}$ in the following two cases:

Case 1: $x_{i}<0$. In this case, for $0 \leq p_{i} \leq$ $\min \left(P_{i}^{\max }, \frac{\bar{Q}-\sum_{j=1, j \neq i}^{N} p_{j} g_{j}}{g_{i}}, \bar{P}-\sum_{j=1, j \neq i}^{N} p_{j}\right)$, we have $\frac{\partial f(\boldsymbol{p})}{\partial p_{i}}>0$ and thus $f(\boldsymbol{p})$ is a increasing function of $p_{i}$. Thus, we can increase $p_{i}$ to $\min \left(P_{i}^{\max }, \frac{\bar{Q}-\sum_{j=1, j \neq i}^{N} p_{j} g_{j}}{g_{i}}, \bar{P}-\right.$ $\left.\sum_{j=1, j \neq i}^{N} p_{j}\right)$ to increase the value of $f(\boldsymbol{p})$. This will make one of the constraints in (41)-(43) become equality constraint. This contradicts with our assumption that the constraints in (41)-(43) are satisfied with strict inequality.

Case 2: $x_{i}>P_{i}^{\max }$. In this case, for $0 \leq p_{i} \leq$ $\min \left(P_{i}^{\max }, \frac{\bar{Q}-\sum_{j=1, j \neq i}^{N} p_{j} g_{j}}{g_{i}}, \bar{P}-\sum_{j=1, j \neq i}^{N} p_{j}\right)$, we have $\frac{\partial f(\boldsymbol{p})}{\partial p_{i}}<0$ and thus $f(\boldsymbol{p})$ is a decreasing function of $p_{i}$. Based on this, we can decrease $p_{i}$ to 0 to increase the value of $f(\boldsymbol{p})$. This will lead to equality constraint in (44) for the arbitrary $i \in\{1, \ldots, N\}$, which contradicts with our assumption that the constraints in (44) are satisfied with strict inequality.

Case 3: $0 \leq x_{i} \leq P_{i}^{\max }$. In this case, we have $\frac{\partial f(\boldsymbol{p})}{\partial p_{i}}<0$ for $0 \leq p_{i}<x_{i}$ and $\frac{\partial f(\boldsymbol{p})}{\partial p_{i}}>0$ for $p_{i}>x_{i}$, and thus $f(\boldsymbol{p})$ first decreases as $p_{i}$ increases for $0 \leq p_{i}<x_{i}$ and then increases as $p_{i}$ increases for $p_{i}>x_{i}$. Thus, the value of $f(\boldsymbol{p})$ can be increased by increasing $p_{i}$ to $\min \left(P_{i}^{\max }, \frac{\bar{Q}-\sum_{j=1, j \neq i}^{N} p_{j} g_{j}}{g_{i}}, \bar{P}-\right.$ $\left.\sum_{j=1, j \neq i}^{N} p_{j}\right)$ or decreasing $p_{i}$ to 0 . This results in one of the constraints in (41)-(44) becoming equality constraint, which contradicts with our assumption that all of the constraints in (41)-(44) are satisfied with strict inequality.

Lemma 5 is thus proved.

Lemma 6: At most one SU's transmit power satisfies $0<$ $p_{i}<P_{i}^{\max }, i \in\{1, \ldots, N\}$ for the optimal solution of $\mathrm{P} 2$.

Proof: If both the two constraints in (41) and (42) are satisfied with strict inequality, then according to Lemma 5, $p_{i}, \forall i$ is either 0 or $P_{i}^{\max }$. Thus, there is no SU's transmit power satisfies $0<p_{i}<P_{i}^{\max }$.

Next, considering the case that at least one of the two constraints in (41) and (42) is satisfied with equality. For arbitrary $x \in\{1, \ldots, N\}$ and $y \in\{1, \ldots, N\}, y \neq x$, we assume that $p_{x}$ and $p_{y}$ satisfies $0<p_{x}<P_{x}^{\max }$ and $0<p_{y}<P_{y}^{\max }$, respectively. First, we consider the case that $h_{x}>h_{y}$ and $\sum_{i=1}^{N} p_{i}=\bar{P}$. Then $p_{y}$ can be written as $p_{y}=\bar{P}-p_{x}-A$, where $A=\sum_{j=1, j \neq x, j \neq y}^{N} p_{i}$. Let $B=\sum_{j=1, j \neq x, j \neq y}^{N} p_{j} h_{j}$ and $C_{i}=\sum_{j=1, j \neq i, j \neq x, j \neq y}^{N} p_{j} h_{j}$. Then, $f(\boldsymbol{p})$ can be rewritten as

$$
\begin{aligned}
& f(\boldsymbol{p})=\sum_{i=1}^{N} \ln \left(1+\frac{p_{i} h_{i}}{\sigma^{2}+\sum_{j=1, j \neq i}^{N} p_{j} h_{j}}\right) \\
& =\ln \left(1+\frac{p_{x} h_{x}}{\sigma^{2}+\left(\bar{P}-p_{x}-A\right) h_{y}+B}\right) \\
& +\ln \left(1+\frac{\left(\bar{P}-p_{x}-A\right) h_{y}}{\sigma^{2}+p_{x} h_{x}+B}\right) \\
& +\sum_{i=1, i \neq x, i \neq y}^{N} \ln \left(1+\frac{p_{i} h_{i}}{\sigma^{2}+p_{x} h_{x}+\left(\bar{P}-p_{x}-A\right) h_{y}+C_{i}}\right)
\end{aligned}
$$

The first derivative of $f(\boldsymbol{p})$ with respect to $p_{x}$ can then be obtained as

$$
\begin{aligned}
& \frac{\partial f(\boldsymbol{p})}{\partial p_{x}}=\frac{1}{\sigma^{2}+B+(\bar{P}-A) h_{y}+\left(h_{x}-h_{y}\right) p_{x}} \\
& \times\left(\frac{\left(\sigma^{2}+B\right) h_{x}+(\bar{P}-A) h_{x} h_{y}}{\sigma^{2}+B+(\bar{P}-A) h_{y}-h_{y} p_{x}}\right. \\
& -\frac{\left(\sigma^{2}+B\right) h_{y}+(\bar{P}-A) h_{x} h_{y}}{\sigma^{2}+B+h_{x} p_{x}} \\
& \left.+\sum_{i=1, i \neq x, i \neq y}^{N} \frac{-p_{i} h_{i}\left(h_{x}-h_{y}\right)}{\sigma^{2}+C_{i}+(\bar{P}-A) h_{y}+\left(h_{x}-h_{y}\right) p_{x}}\right) .
\end{aligned}
$$

It is observed that, since $h_{x}>h_{y}, 1 / \sigma^{2}+B+(\bar{P}-A) h_{y}+$ $\left(h_{x}-h_{y}\right) p_{x}$ is positive, and the remaining part of $\frac{\partial f(\boldsymbol{p})}{\partial p_{x}}$ is a increasing function of $p_{x}$. Thus, the solution to $\frac{\partial f(\boldsymbol{p})}{\partial p_{x}}=0$ is unique, and is denoted as $z$. It is easy to observed that $\left.\frac{\partial f(\boldsymbol{p})}{\partial p_{x}}\right|_{p_{x}=0}<0$. Thus, we have $z>0$. We discuss the optimal $p_{x}$ in the following three cases:

Case 1: $z>P_{x}^{\max }$. In this case, $f(\boldsymbol{p})$ is a decreasing function of $p_{x}$ for $0 \leq p_{x} \leq P_{x}^{\max }$. Thus, we can decrease $p_{x}$ to 0 to increase the value of $f(\boldsymbol{p})$. This contradicts with our assumption that $0<p_{x}<P_{x}^{\max }$.

Case 2: $0<z \leq P_{x}^{\max }$. In this case, $f(\boldsymbol{p})$ first decreases as $p_{x}$ increases for $0 \leq p_{x}<z$ and then increases as $p_{x}$ increases for $p_{x}>z$. Thus, the value of $f(\boldsymbol{p})$ can be increased by increasing $p_{i}$ to $P_{x}^{\max }$ or decreasing $p_{i}$ to 0 . This contradicts with our assumption that $0<p_{x}<P_{x}^{\max }$.

For the other cases such as $h_{y}>h_{x}$ and $\sum_{i=1}^{N} p_{i} g_{i}=\bar{Q}$, we can write $p_{x}=\frac{\bar{Q}-p_{y} g_{y}-\sum_{i \neq x, i \neq y}^{N} p_{i} g_{i}}{\text { and use the same }}$ method to prove that $0<p_{y}<^{g_{x}} P_{y}^{\max }$ is not optimal.

Lemma 6 is thus proved

Based on Lemma 5 and Lemma 6, we propose a heuristic algorithm to solve P2 as shown in Algorithm 2.

\section{Simulation Results}

In this section, simulations are presented to verify the performance of the proposed algorithms. All the channels involved are assumed to follow Rayleigh fading with unit mean. The noise power is set to 1 . For simplicity, the peak 

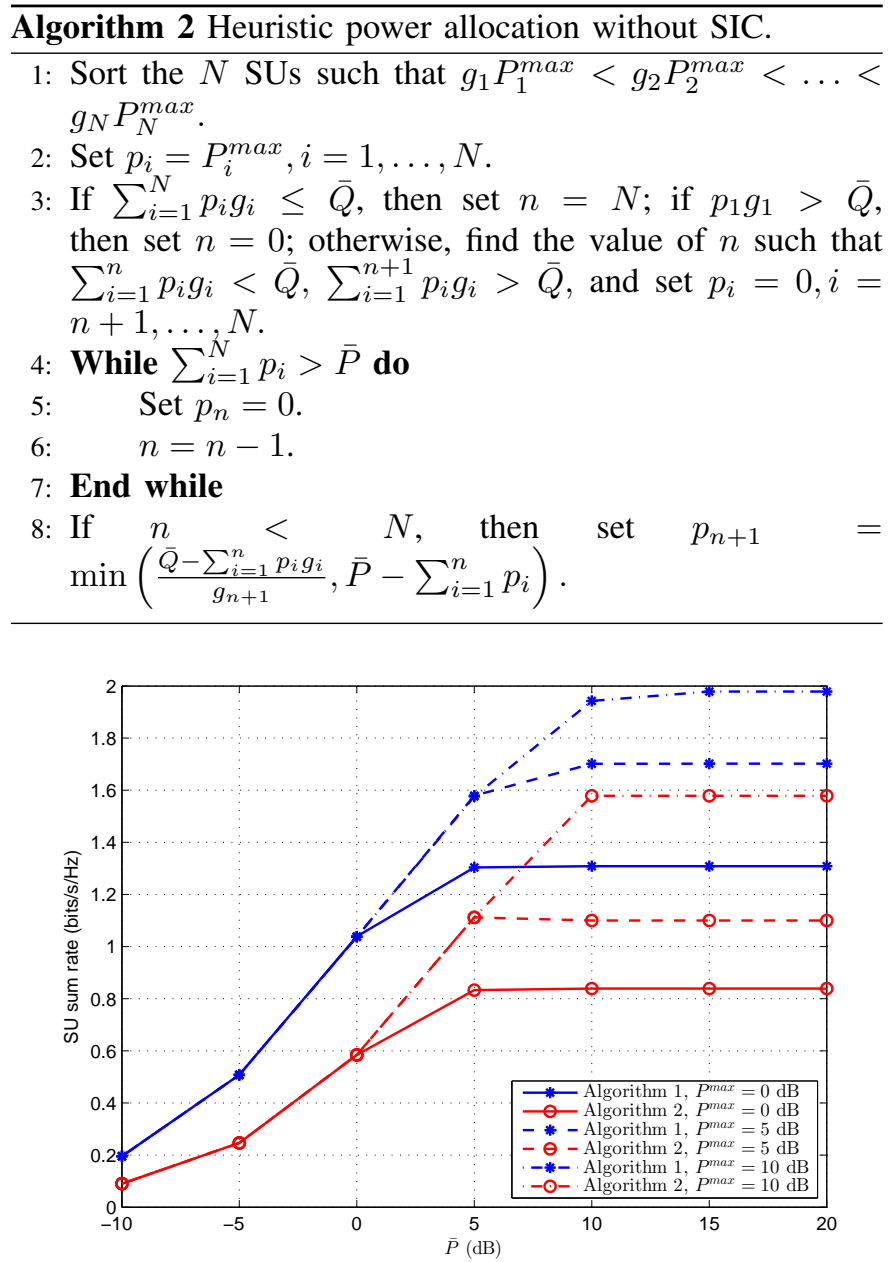

Fig. 2. SU sum rate against $\bar{P}(N=5, \bar{Q}=0 \mathrm{~dB})$.

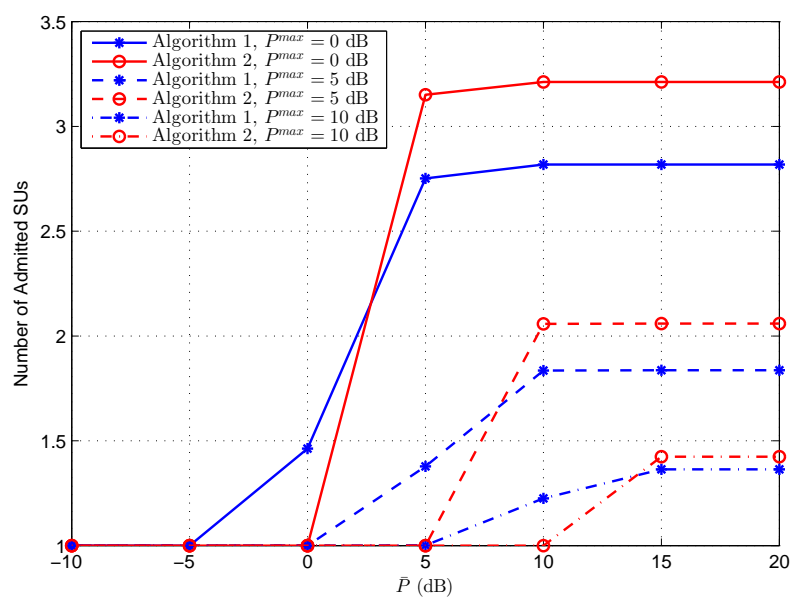

Fig. 3. Number of admitted SUs against $\bar{P}(N=5, \bar{Q}=0 \mathrm{~dB})$.

transmit power limits of all the SUs are assumed to be the same and denoted as $P^{\max }$. The following results are obtained by averaging over 1000 simulation runs.

Figs. 2 and 3 plot the SU sum rate and the number of admitted SUs, respectively, against $\bar{P}$ under various values of $P^{\max }$. It is noted that the admitted SUs' transmit powers are nonzero. It is seen that the SU sum rate achieved by Algorithm

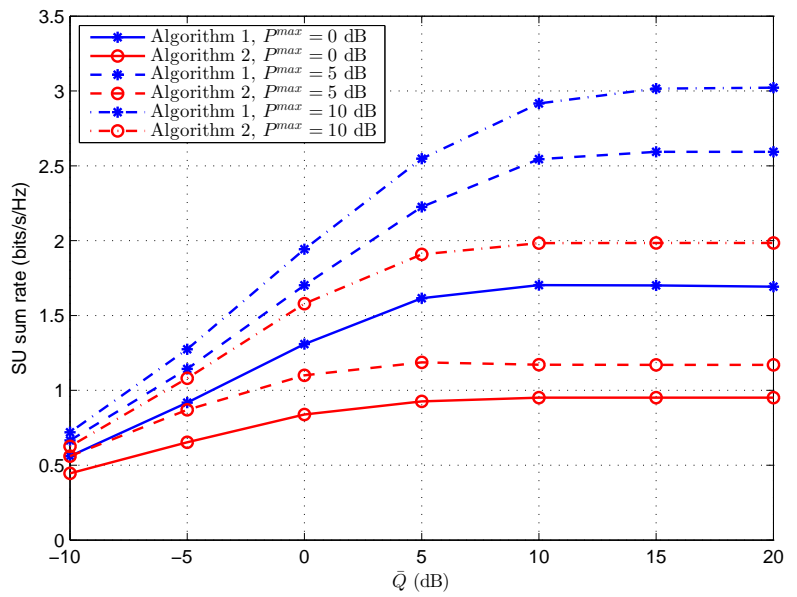

Fig. 4. SU sum rate against $\bar{Q}(N=5, \bar{P}=10 \mathrm{~dB})$.

1 with SIC is always higher than that achieved by Algorithm 2 without SIC. This is due to the fact that SIC can cancel mutual interference between the SUs and thus enhance the performance of the SUs. It is also seen that the SU sum rates under various values of $P^{\max }$ are the same and increase as $\bar{P}$ increases for a small value of $\bar{P}$, and then saturate to different levels for a high value of $\bar{P}$. This is due to the fact that the SU performance is limited by $\bar{P}$ for a small value of $\bar{P}$ and increasing the value of $P^{\max }$ does not improve the SU sum rate. It is seen that higher $P^{\max }$ results in higher saturated SU sum rate. In addition, it is observed that the number of admitted SUs increases as $\bar{P}$ increases and then keep constant for a high value of $\bar{P}$. This is because that increasing $\bar{P}$ results in more transmit power that can be allocated to the SUs for a small value of $\bar{P}$. It is also observed that, compared to the number of admitted SUs achieved by Algorithm 2, Algorithm 1 achieves higher number of admitted SUs when $\bar{P}$ is medium and achieves lower number of admitted SUs when $\bar{P}$ is high. It is seen that such transition point of $\bar{P}$ decreases as $P^{\max }$ decreases. This indicates that, although Algorithm 2 without SIC performs worse than Algorithm 1 with SIC in terms of the SU sum rate, it can admit more number of SUs for a large $\bar{P}$. Furthermore, it is seen that a lower value of $P^{\max }$ leads to larger number of admitted SUs. This is due to the fact that a lower $P^{\max }$ will cause the SU with better channel condition to consume less transmit power and thus more SUs can be admitted.

Figs. 4 and 5 plot the SU sum rate and the number of admitted SUs, respectively, against $\bar{Q}$ under various values of $P^{\max }$. Similar to Fig. 2, it is seen that Algorithm 1 achieves higher SU sum rate than that of Algorithm 2. It is also seen that the SU sum rates under various values of $P^{\max }$ are different and increase as $\bar{Q}$ increases and then saturate to different values for a high value of $\bar{Q}$. This is because that the performance of the SUs is limited by $\bar{Q}$ for a small value of $\bar{Q}$ and increasing the value of $\bar{Q}$ can improve the SU sum rate, while for a large value of $\bar{Q}$, the performance of the SUs is limited by $\bar{P}$ and increasing the value of $\bar{Q}$ can not improve the SU performance. In addition, it is observed that the number of admitted SUs increases as $\bar{Q}$ increases and then 


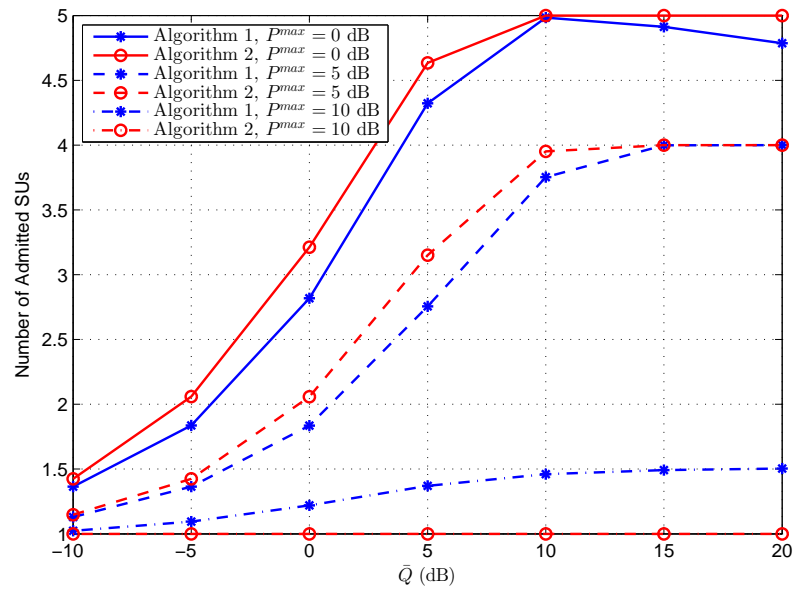

Fig. 5. Number of admitted SUs against $\bar{Q}(N=5, \bar{P}=10 \mathrm{~dB})$.

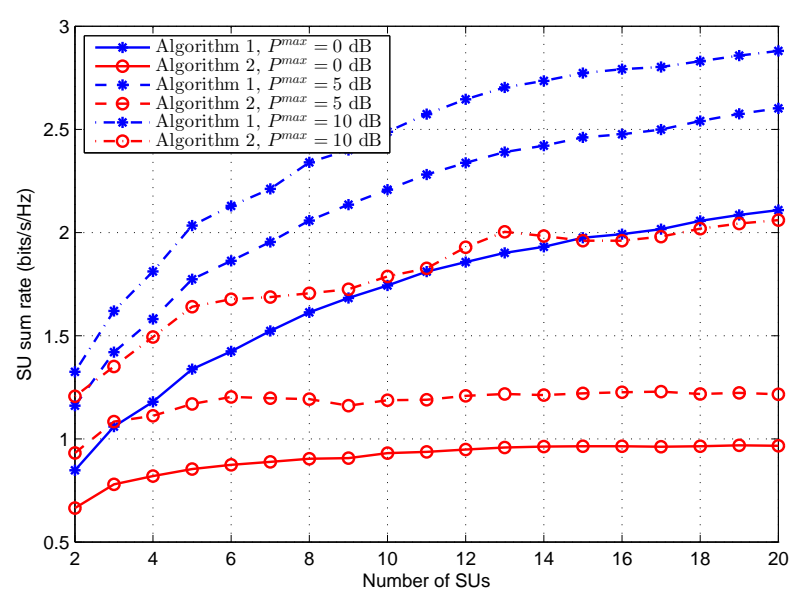

Fig. 6. SU sum rate against $N(\bar{P}=10 \mathrm{~dB}, \bar{Q}=0 \mathrm{~dB})$.

keep constant in most cases for a high value of $\bar{Q}$. This is owning to the fact that increasing $\bar{Q}$ results in more transmit power that can be allocated to the SUs for a small value of $\bar{Q}$, while for a large $\bar{Q}, \bar{P}$ restricts the transmit power of the SUs and thus increasing $\bar{Q}$ can not increase the number of admitted SUs. Furthermore, it is observed that Algorithm 2 achieves lower number of admitted SUs than that of Algorithm 1 when $P^{\max }$ is high, and achieves higher number of admitted SUs than that of Algorithm 1 when $P^{\max }$ is low. This indicates that, Algorithm 2 without SIC performs better than Algorithm 1 with SIC in terms of the number of admitted SUs for a small $P^{\max }$.

Figs. 6 and 7 plot the SU sum rate and the number of admitted SUs, respectively, against the number of SUs $N$ under various values of $P^{\max }$. It is seen that the $\mathrm{SU}$ sum rate increases with the increase of $N$. This is because that a larger value of $N$ results in higher probability that more SUs are in good channel conditions which leads to higher SU sum rate. It is also seen that the impact of increasing $N$ on the $\mathrm{SU}$ sum rate for a large $N$ is weaker than that for a small $N$. In addition, It is seen that the number of admitted SUs increases as $N$ increases except the case that $P^{\max }$ is very large for Algorithm 2. This may be due to the fact that a larger value of $N$ leads to higher probability that more SUs are in good

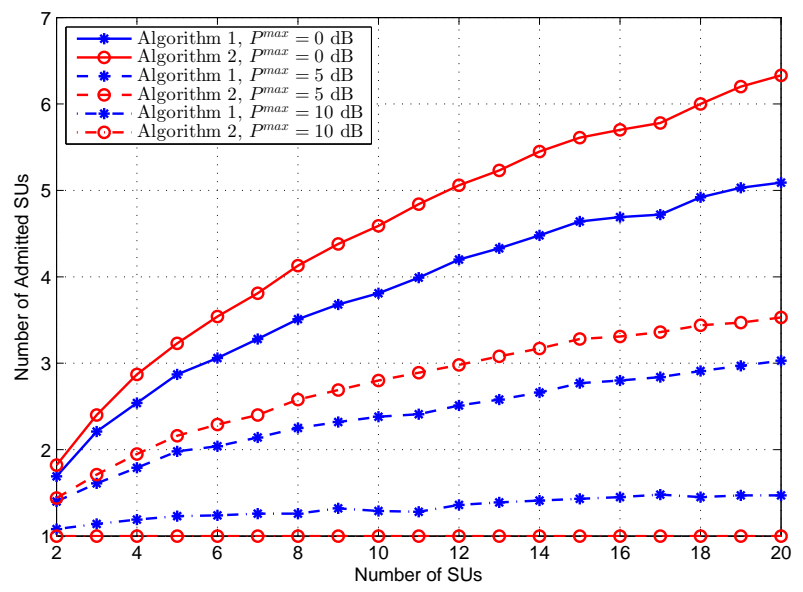

Fig. 7. Number of admitted SUs against $N(\bar{P}=10 \mathrm{~dB}, \bar{Q}=0 \mathrm{~dB})$.

channel conditions which results in higher number of admitted SUs, while for a very large $P^{\max }$, there may exist some SUs with good channel conditions that consume all the transmit power resources which results in constant number of admitted SUs.

\section{CONCLUSions}

We consider a fading CMAC with a power station harvesting energy from the nature and then providing power to the SUs. The problems of power allocation for such a CMAC to maximize the SU sum rate under the interference power constraint, the sum transmit power constraint and the peak transmit power constraint of each individual SU are investigated for two scenarios: with SIC and without SIC. The optimal power allocation algorithm for the first scenario and a heuristic algorithm for the second scenario are proposed respectively. It is shown that the proposed algorithm with SIC achieves higher SU sum rate than that of the algorithm without SIC, while the number of admitted SUs achieved by the algorithm without SIC is higher than that achieved by the algorithm with SIC for a high sum transmit power limit and a low peak transmit power limit of each individual SU. It is also shown that the number of admitted SUs increases with the decrease of the peak transmit power limit of each individual SU.

\section{REFERENCES}

[1] J. Xue, Z. Feng, and K. Chen, "Beijing spectrum survey for cognitive radio applications," in IEEE Vehicular Technology Conference Fall, 2013, pp. $1-5$.

[2] S. Haykin, "Cognitive radio: brain-empowered wireless communications," IEEE Journal on Selected Areas in Communications, vol. 23, no. 2, pp. 201-220, 2005.

[3] X. Kang, Y.-C. Liang, A. Nallanathan, K. Garg, and R. Zhang, "Optimal power allocation for fading channels in cognitive radio networks: Ergodic capacity and outage capacity," IEEE Transactions on Wireless Communications, vol. 8, no. 2, pp. 940-950, 2009.

[4] L. Qun and X. Ding, "Power allocation for ergodic capacity and outage probability tradeoff in cognitive radio networks," IEICE Transactions on Communications, vol. 98, no. 10, pp. 1988-1995, 2015.

[5] R. Zhang, S. Cui, and Y.-C. Liang, "On ergodic sum capacity of fading cognitive multiple-access and broadcast channels," IEEE Transactions on Information Theory, vol. 55, no. 11, pp. 5161-5178, 2009. 
[6] X. Kang, Y.-C. Liang, and H. K. Garg, "Fading cognitive multiple access channels: Outage capacity regions and optimal power allocation," IEEE Transactions on Wireless Communications, vol. 9, no. 7, pp. 2382-2391, 2010.

[7] W. Wang, W. Wang, Q. Lu, K. G. Shin, and T. Peng, "Geometry-based optimal power control of fading multiple access channels for maximum sum-rate in cognitive radio networks," IEEE Transactions on Wireless Communications, vol. 9, no. 6, pp. 1843-1848, 2010.

[8] D. Li and Y.-C. Liang, "Power allocation for interference-limited cognitive multiple access channels," IEEE Wireless Communications Letters, vol. 2, no. 3, pp. 291-294, 2013.

9] L. Zhang, Y. Xin, Y.-C. Liang, and H. V. Poor, "Cognitive multiple access channels: optimal power allocation for weighted sum rate maximization," IEEE Transactions on Communications, vol. 57, no. 9, pp. 2754-2762, 2009.

[10] X. Kang, "Optimal power allocation for fading cognitive multiple access channels: a two-user case," IEEE Wireless Communications Letters, vol. 2, no. 6, pp. 683-686, 2013.

[11] D. Xu and Q. Li, "On the effective capacity region for cognitive radio multiple access channels," AEU-International Journal of Electronics and Communications, vol. 69, no. 6, pp. 958-961, 2015.
[12] X. Kang, H. F. Chong, Y.-K. Chia, and S. Sun, "Ergodic sum-rate maximization for fading cognitive multiple-access channels without successive interference cancelation," IEEE Transactions on Vehicular Technology, vol. 64, no. 9, pp. 4009-4018, 2015.

[13] D. Xu and Q. Li, "Power allocation for two-user cognitive multiple access channels under primary user outage constraint," International Journal of Communication Systems, to be published.

[14] W. Chung, S. Park, S. Lim, and D. Hong, "Optimal transmit power control for energy-harvesting cognitive radio system," in IEEE Vehicular Technology Conference Fall, 2013, pp. 1-5.

[15] V. Rakovic, D. Denkovski, Z. Hadzi-Velkov, and L. Gavrilovska, "Optimal time sharing in underlay cognitive radio systems with RF energy harvesting," in IEEE International Conference on Communications, 2015, pp. 7689-7694.

[16] D. T. Hoang, D. Niyato, P. Wang, and D. I. Kim, "Opportunistic channel access and RF energy harvesting in cognitive radio networks," IEEE Journal on Selected Areas in Communications, vol. 32, no. 11, pp. 2039 2052, 2014

[17] S. Boyd and L. Vandenberghe, Convex Optimization. Cambridge, U.K.: Cambridge Univ. Press, 2004. 